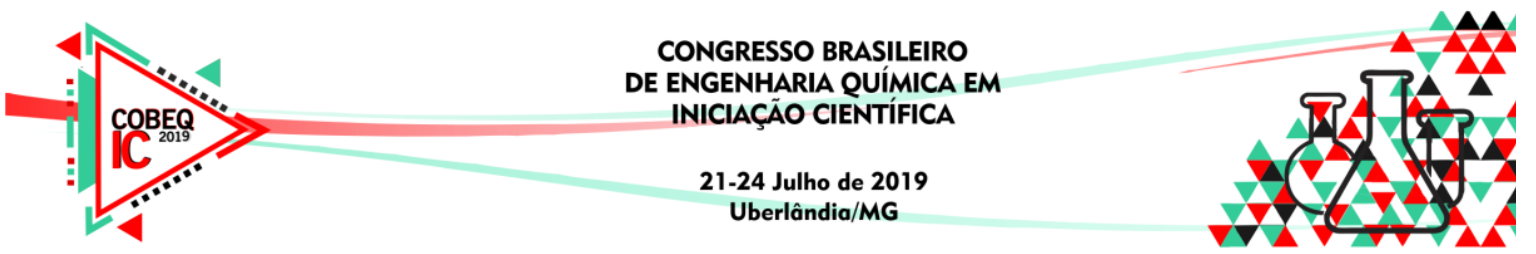

\title{
SÍNTESE DE COMPÓSITOS HDL-BIOCARVÃO DE OURICURI PARA APLICAÇÃO NA REMOÇÃO DE POLUENTES EMERGENTES
}

\author{
G. E. S. SANTOS ${ }^{1}$, Í. M. G. L. SÁ ${ }^{2}$, L.L.I. SILVA ${ }^{2}$ e L. MEILI ${ }^{2}$ \\ ${ }^{1}$ Universidade Federal de Uberlândia, Faculdade de Engenharia Química \\ ${ }^{2}$ Universidade Federal de Alagoas, Centro de Tecnologia \\ E-mail para contato: grazi.gess@ hotmail.com
}

\begin{abstract}
RESUMO - O presente trabalho teve como objetivo avaliar a capacidade adsortiva do HDL-biocarvão para remoção de poluentes como cafeína, diclofenaco de sódio e fenol. O compósito foi sintetizado pelo método da co-precipitação tendo $\mathrm{Mg}$ e $\mathrm{Al}$ como cátions presentes nas lamelas, intercalados pelo ânion cloreto. A partir dos estudos de adsorção, observou-se que o compósito conseguiu adsorver o diclofenaco de sódio, alcançando patamares de $66 \%$ de remoção. Para a cafeína e fenol o compósito não apresentou afinidade de modo que não ocorreu remoção. $\mathrm{O}$ estudo cinético mostrou que o equilíbrio de adsorção ocorreu em uma hora de contato para o diclofenaco de sódio com concentração de $30 \mathrm{mg} / \mathrm{L}$.
\end{abstract}

\section{INTRODUÇÃO}

Diversos produtos químicos são utilizados na sociedade moderna, produzidos mundialmente em larga escala. Devido a essa produção e utilização, ocorre a geração de diversos resíduos, que são derivados diretamente das atividades industriais ou pelo consumo da sociedade. Diante disso, diversos estudos demonstram uma preocupação relacionada aos impactos ambientais causados pela presença de novos compostos em águas residuais, subterrâneas e superficiais (Silva e Collins, 2011). Os poluentes emergentes são compostos químicos naturais ou sintéticos que apresentam ameaças potenciais aos ecossistemas ambientais e à saúde humana. Apesar de sua presença na natureza não ser recente, esses poluentes têm se destacado nas últimas décadas, devido ao desenvolvimento de tecnologias e métodos de detecção analíticas. Esses contaminantes estão presentes em uma grande variedade de produtos comerciais, tais como, produtos farmacêuticos, inseticidas, aditivos alimentares entre outros (Barceló, 2003).

Os fármacos, embora sejam encontrados em pequenas quantidades, vêm causando diversas preocupações devido a sua contribuição continua ao meio ambiente. Além disso, os sistemas convencionais de tratamento não são eficientes na remoção desses poluentes. $O$ diclofenaco de sódio é um anti-inflamatório não esteroidal sintético (AINE), está entre os medicamentos mais prescritos no mundo devido ao seu uso em tratamentos medicinais, como analgésico, antiartrítico e antirreumático (Zhang et al., 2011). Já a cafeína é um ingrediente muito utilizado em medicamentos, frequentemente combinado com outros medicamentos como analgésicos e anti-histamínicos (International, 1991). O fenol é um dos poluentes orgânicos mais perigosos presentes nas águas residuais devido a sua alta toxidade. Sua presença ocorre 


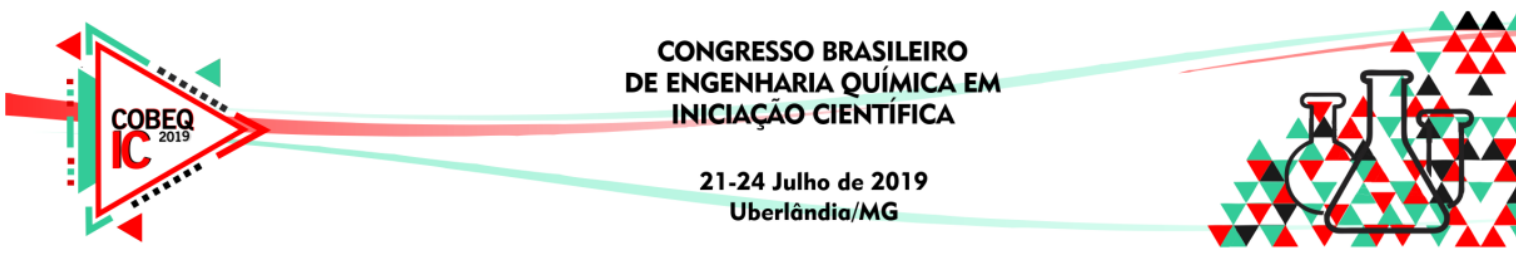

por diversas atividades industriais como mineração, refino de petróleo e produção farmacêutica (Mohammadi et al., 2014).

Vários métodos são propostos para remoção de poluentes orgânicos e inorgânicos, incluído o processo de adsorção. Pois é um processo eficiente, com baixo consumo energético e sem a adição de subprodutos indesejáveis (Wang et al., 2015). A adsorção empregando biocarvões tem se mostrado como uma alternativa devido ele ser proveniente de uma fonte renovável, além de transformar a biomassa em um produto com maior valor agregado (Mohan et al. 2006). A utilização de hidróxidos duplos lamelares (HDLs) suportados em biocarvões tem aumentado a eficiência no processo de adsorção para a remoção de poluentes (Zhang et al. 2014). Desse modo, este trabalho tem como objetivo sintetizar compósitos de HDL-biocarvão a fim de validá-los como adsorventes no tratamento de água contaminada com efluentes como a cafeína, diclofenaco de sódio e fenol.

\section{METODOLOGIA}

O biocarvão foi produzido realizando a pirólise do endocarpo de ouricuri utilizando um forno tubular aquecido por resistências, com sistema de resfriamento, controlador de temperatura e taxa de aquecimento. $O$ processo foi realizado à vácuo com o tempo de residência de $120 \mathrm{~min}$, temperatura final de $400^{\circ} \mathrm{C}$ e taxa de aquecimento de $10^{\circ} \mathrm{C} / \mathrm{min}$. O biocarvão obtido foi padronizado para a granulometria de 28 a 65 mesh. A síntese do compósito foi realizada pelo método de co-precipitação com uma proporção de 2:1 de magnésio e alumínio. Foram pesados $18,1 \mathrm{~g}$ de cloreto de alumínio e $30,4 \mathrm{~g}$ de cloreto de magnésio e adicionado $120 \mathrm{~mL}$ de água deionizada. Os reagentes foram mantidos sob agitação por 30 minutos para completa dissolução dos componentes. Foi adicionado à solução $5 \mathrm{~g}$ de biocarvão e iniciou-se o gotejamento com solução de hidróxido de sódio $3 \mathrm{~mol} / \mathrm{L}$ até alcançar o $\mathrm{pH} 10$. O ajuste do $\mathrm{pH}$ foi realizado em duas horas e a mistura permaneceu sob agitação por mais duas horas. Posteriormente, a solução foi centrifugada por cinco minutos à 3000rpm, a fase líquida foi retirada e a fase sólida foi lavada com água deionizada até o $\mathrm{pH}$ da água ficar em torno de 7. Por fim, o material foi depositado em placas de petri, seguindo para a estufa, onde permanece por 18 horas a $60^{\circ} \mathrm{C}$. O compósito HDL-Biocarvão foi triturado e padronizado para o tamanho de partícula na faixa entre 0,208 e $0,589 \mathrm{~mm}$.

As soluções estoque de cafeína, diclofenaco de sódio e fenol foram preparadas com concentração de $1000 \mathrm{mg} / \mathrm{L}$ utilizando água deionizada $(18,2 \mathrm{M} \Omega)$. Para determinação do comprimento de onda, registrou-se um espectro de absorção na faixa de 220 a 320nm para as soluções foram diluídas nas concentrações de 5,10,20,30 e 50mg/L. A curva analítica foi construída medido no comprimento de onda máximo as soluções com concentrações conhecidas de cafeína, diclofenaco de sódio e fenol.

Para avaliar a aplicabilidade do adsorvente na remoção dos adsorbatos, foi realizado um teste de afinidade. $\mathrm{O}$ teste de afinidade foi conduzido em duplicata dispondo $0,1 \mathrm{~g}$ de adsorvente em erlenmeyer junto com uma solução de $25 \mathrm{~mL}$ de adsorbato a $30 \mathrm{ppm}$. As amostras foram dispostas em agitador orbital sob rotação de $140 \mathrm{rpm}$, temperatura de $30^{\circ} \mathrm{C}$ por um tempo de 24 horas. Em seguida, foi realizada a leitura das absorbâncias das amostras num espectrofotômetro com comprimento de onda correspondente a solução obtida previamente através de dados experimentais. A quantidade adsorvida foi calculada de acordo com a Equações (1). 


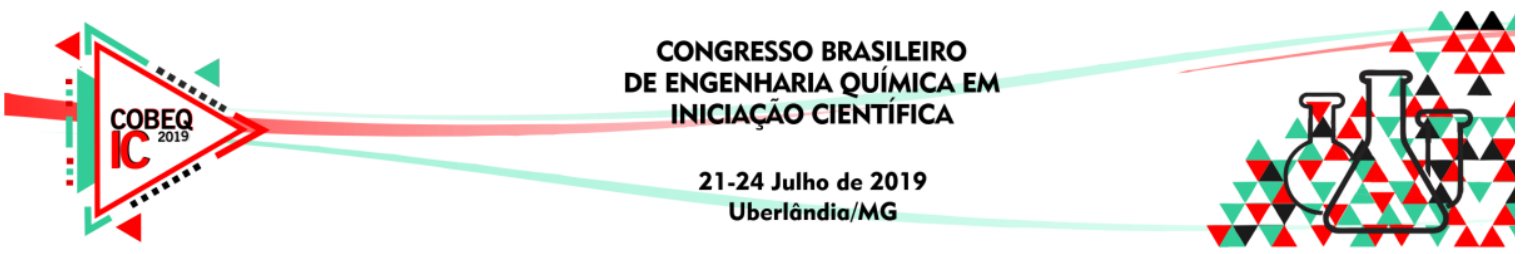

$\mathrm{q}_{\mathrm{t}}=\frac{\mathrm{C}_{\mathrm{f}}-\mathrm{C}_{0}}{\mathrm{~m}} * \mathrm{~V}$

onde $q_{t}$ é a quantidade adsorvida (mg/g) em um tempo $t$, $\mathrm{C}_{\mathrm{f}}$ é a concentração final, $\mathrm{C}_{0}$ a concentração inicial, $m$ massa do adsorvente e $V$ o volume do adsorbato.

Para o estudo da cinética de adsorção, foram utilizados os mesmos parâmetros, contudo variou-se o tempo em 5, 10, 15, 30, 60, 120 e 240 minutos. Os dados obtidos foram ajustados para os modelos matemáticos de pseudo-primeira ordem (Equação 2) e pseudo-segunda ordem (Equação 3) de modo a descrever o comportamento da cinética de adsorção (Lagergren, 1898; Ho, 2000).

$\mathrm{q}_{\mathrm{t}}=\mathrm{q}_{\mathrm{e}}\left[1-\exp \left(-\mathrm{k}_{1} * \mathrm{t}\right)\right]$

$q_{t}=\frac{k_{2} q_{e}^{2} t}{1+k_{2} q_{e} t}$

onde $q_{e}$ é quantidade adsorvida $(\mathrm{mg} / \mathrm{g})$ no equilíbrio, $k_{l}$ é a constante de velocidade de pseudo primeira ordem $\left(\mathrm{min}^{-1}\right)$ e $k_{2}$ é a constante de velocidade de pseudo segunda ordem (g/mg.min).

\section{RESULTADOS E DISCUSSÕES}

O HDL-Biocarvão sintetizado pode ser visto na Figuras 1, em que aparece, respectivamente, o compósito antes da secagem e o compósito após a secagem.

Figura 1 - Compósito HDL-Biocarvão antes da secagem (a) e após a secagem (b)
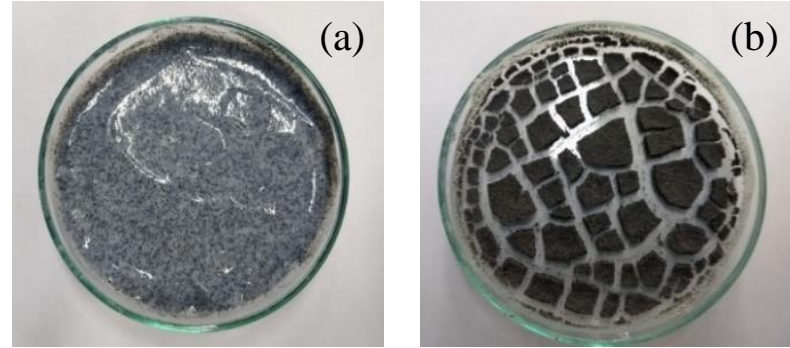

Para verificar a composição do material sintetizado, foi realizado a técnica de EDX. De modo a quantificar os elementos presentes na amostra por fluorescência de raios $\mathrm{X}$ por energia dispersiva. A análise consiste na excitação dos elementos que constituem a amostra, assim emitem linhas espectrais com energia característica do elemento e cuja a intensidade está relacionada com a concentração do elemento na amostra, podendo assim serem detectados (Nascimento Filho, 1997). Com isso, realizou-se a análise e verificou que houve a presença de $34 \%$ de magnésio, $20 \%$ de alumínio e $44 \%$ de cloro. Comprovando que o material sintetizado apresentou os cátions de $\mathrm{Mg}$ e $\mathrm{Al}$, presentes nas lamelas do HDL, e o ânion cloreto intercalado. O que sugere que houve a formação do compósito HDL-biocarvão.

A Figura 2 mostram os espectros com as leituras de absorbância para as soluções de cafeína, diclofenaco de sódio e fenol. Com isso é possível observar o comportamento das soluções e determinar o comprimento de onda máximo. 


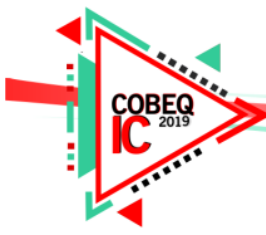

Figura 2 - Espectro com as leituras de absorbância da Cafeína (a), Diclofenaco (b) e Fenol (c)
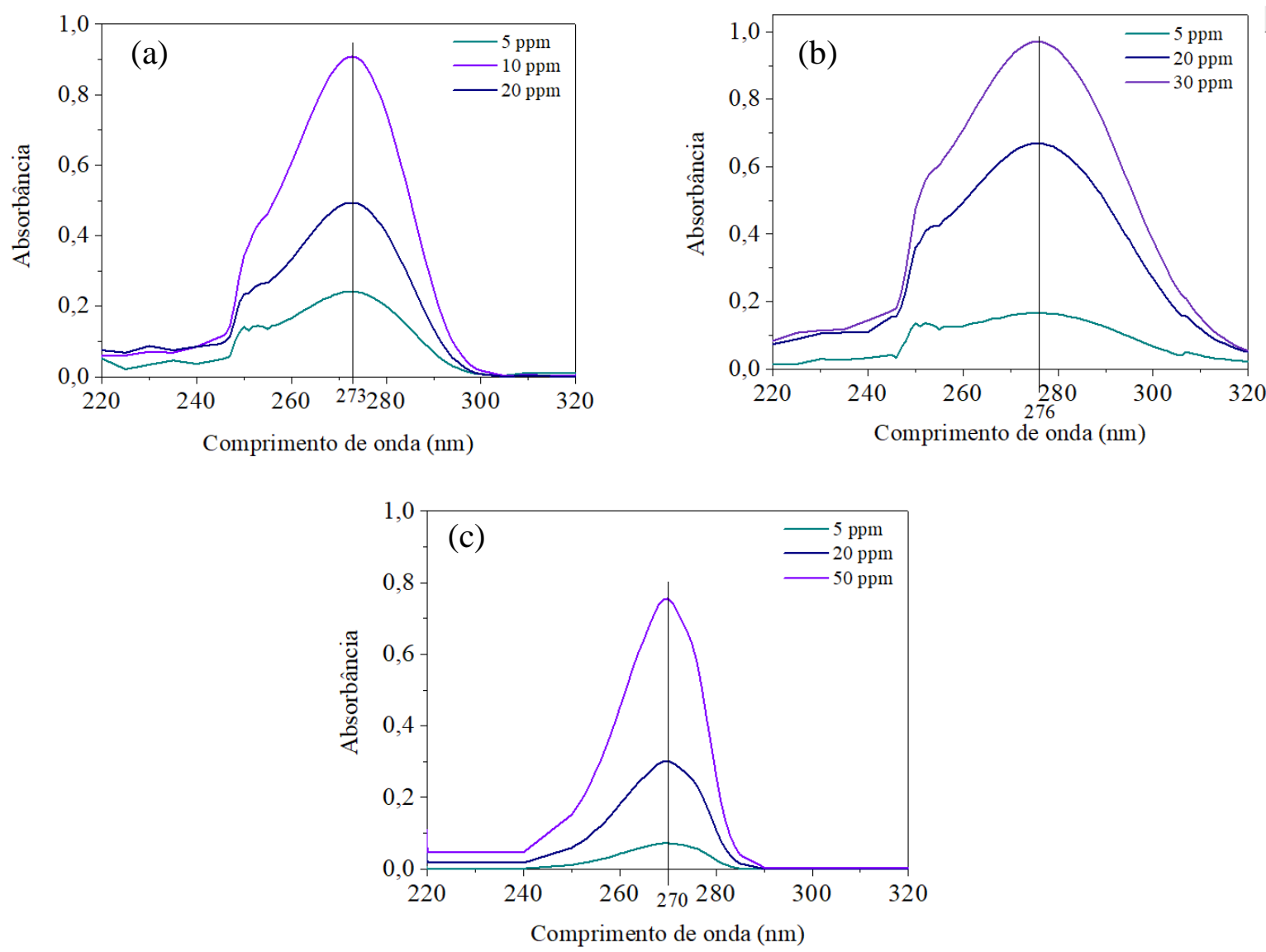

O comprimento de onda utilizado para realização das leituras foi de $273 \mathrm{~nm}$ para cafeína, $276 \mathrm{~nm}$ para o diclofenaco de sódio e $270 \mathrm{~nm}$ para o fenol. Valores condizentes com a literatura, visto que Elhalil et al. (2018), Franco et al. (2018) e Santos et al. (2014), correspondem ao mesmo comprimento de onda para cafeína, para o diclofenaco de sódio e para o fenol, respectivamente. A curva analítica foi determinada utilizando o comprimento de onda máximo para cada soluções e com concentrações conhecidas até obter uma absorbância máxima de um obedecendo os limites exigidos pela Lei de Lambert-Beer. Os valores de absorbância estão dispostos na Tabela 1.

Tabela 1 - Concentrações das soluções dos adsorbatos e respectivas absorbâncias

\begin{tabular}{|c|c|c|c|c|c|}
\hline \multicolumn{2}{|c|}{ Caféna } & \multicolumn{2}{c|}{ Diclofenaco de sódio } & \multicolumn{2}{c|}{ Fenol } \\
\hline $\begin{array}{c}\text { Concentração } \\
(\mathrm{mg} / \mathrm{L})\end{array}$ & Absorbância & $\begin{array}{c}\text { Concentração } \\
(\mathrm{mg} / \mathrm{L})\end{array}$ & Absorbância & $\begin{array}{c}\text { Concentração } \\
(\mathrm{mg} / \mathrm{L})\end{array}$ & Absorbância \\
\hline 0,5 & 0,026 & 0,5 & 0,020 & 2 & 0,030 \\
\hline 1 & 0,051 & 1 & 0,038 & 3 & 0,044 \\
\hline 2 & 0,108 & 2 & 0,070 & 5 & 0,075 \\
\hline 5 & 0,243 & 5 & 0,166 & 20 & 0,304 \\
\hline 10 & 0,501 & 10 & 0,325 & 30 & 0,457 \\
\hline 20 & 0,916 & 20 & 0,657 & 40 & 0,611 \\
\hline- & - & 30 & 0,963 & 50 & 0,761 \\
\hline
\end{tabular}




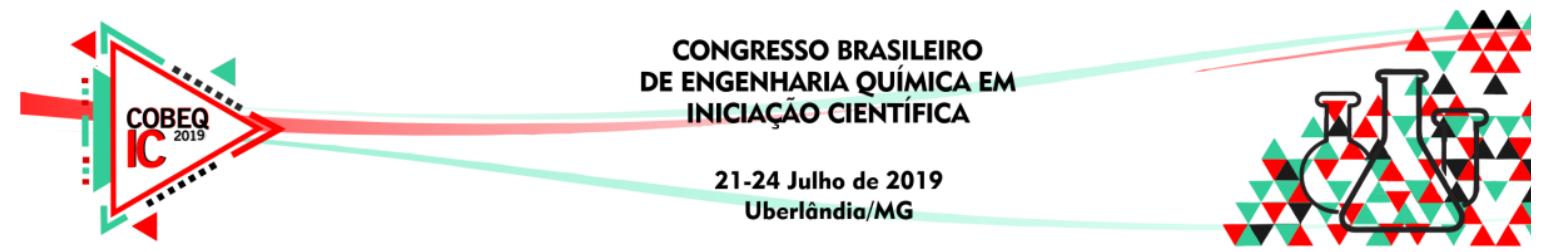

A Lei de Lambert-Beer relaciona a absorção de luz às propriedades do material, e define que a seção transversal de absorção tem que ser constante ao longo de um intervalo de comprimento de onda. Dessa forma, pode-se gerar uma relação linear de absorbância pela concentração (Skoog et al., 2006). Com os dados presentes na Tabela 1 foi obtido a relação linear para os poluentes estudados e construído as curvas analíticas representadas da Tabela 2 juntamente com os resultados do teste de afinidade.

Tabela 2 - Curva analítica e teste de afinidade para os diferentes adsorbatos

\begin{tabular}{|c|c|c|c|}
\hline & Cafeína & Diclofenaco de sódio & Fenol \\
\hline Curva Analitíca & $21,763 \mathrm{x}-0,275$ & $31,155 \mathrm{x}-0,179$ & $65,505 \mathrm{x}+0,074$ \\
\hline $\mathrm{R}^{2}$ & 0,9974 & 0,9998 & 0,9999 \\
\hline Remoção $(\%)$ & - & 66 & - \\
\hline $\mathrm{q}_{\mathrm{t}}(\mathrm{mg} /)$ & - & 5,74 & - \\
\hline
\end{tabular}

Como pode ser observado, as curvas analíticas apresentaram bom ajuste de acordo com o valor do coeficiente linear, pois ambos se aproximaram do unitário. Com isso, foi realizado o teste de adsorção e observou que apenas o diclofenaco de sódio apresentou boa afinidade com o compósito HDL-biocarvão. Desse modo foi realizado a cinética do diclofenaco de sódio, que está representado na Figura 3, para evidenciar o efeito do tempo de contato.

Figura 3 - Dados experimentais ajustados aos modelos cinéticos

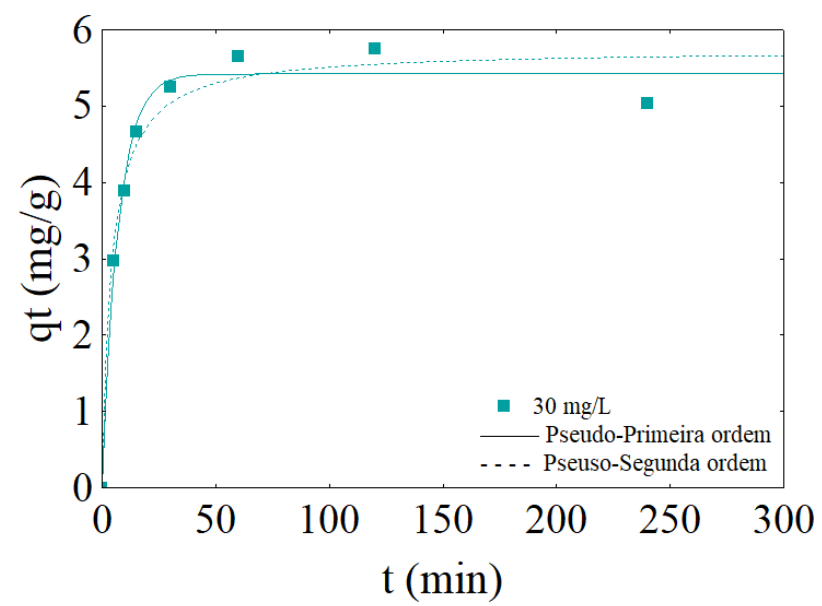

É verificado que o diclofenaco de sódio apresentou equilíbrio no tempo de 120min e obteve porcentagem de remoção $66,51 \%$ com qe de $5,743 \mathrm{mg} / \mathrm{g}$. Pelos ajustes matemáticos, é observado que ambos os modelos são representativos para descrever o comportamento da cinética de adsorção. Além de apresentam coeficiente de correlação aproximadamente 1 e um baixo erro médio relativo. Os valores obtidos pelos ajustes matemáticos estão apresentados na Tabela 3.

Tabela 3 - Parâmetros cinéticos da adsorção do diclofenaco de sódio

\begin{tabular}{|c|c|c|c|c|c|}
\hline \multicolumn{3}{|c|}{ Pseudo-primeira Ordem } & \multicolumn{3}{c|}{ Pseudo-segunda Ordem } \\
\hline $\mathrm{R}^{2}$ & $\mathrm{q}_{\mathrm{e}}(\mathrm{mg} / \mathrm{g})$ & Erro médio Relativo $(\%)$ & $\mathrm{R}^{2}$ & $\mathrm{q}_{\mathrm{e}}(\mathrm{mg} / \mathrm{g})$ & Erro médio Relativo $(\%)$ \\
\hline 0,9839 & 5,42 & 4,83 & 0,9764 & 5,74 & 5,74 \\
\hline
\end{tabular}




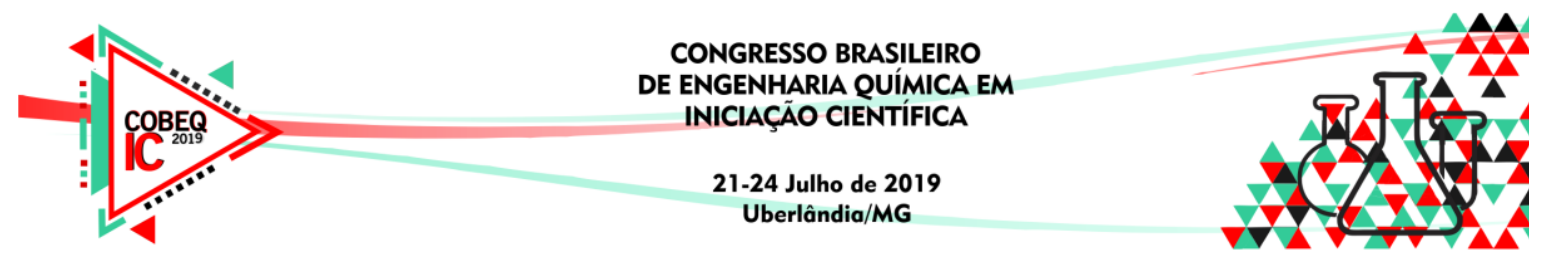

\section{CONCLUSÕES}

Neste trabalho foi sintetizado HDL-biocarvão com o objetivo de adsorver poluentes como cafeína, diclofenaco de sódio e fenol. A análise de EDX verificou que a presença dos componentes de $\mathrm{Mg}$ e $\mathrm{Al}$, cátions presentes nas lamelas, e o ânion cloreto. Já pelo estudo de adsorção, verificou que apenas o diclofenaco apresentou afinidade com o compósito atingindo uma remoção de $66 \%$ em um tempo de 120 minutos.

\section{REFERÊNCIAS}

BARCELÓ, D.,Emerging pollutants in water analysis.Trends Anal.Chem., 22: XIV-XVI. 2003.

ELHALIL, A. et al. Photocatalytic degradation of caffeine as a model pharmaceutical pollutant on $\mathrm{Mg}$ doped $\mathrm{ZnO}-\mathrm{Al} 2 \mathrm{O} 3$ heterostructure. Environmental Nanotechnology, Monitoring \& Management, v. 10, p.63-72, dez. 2018.

FRANCO, M. A. E. de et al. Diclofenac removal from water by adsorption using activated carbon in batch mode and fixed-bed column: Isotherms, thermodynamic study and breakthrough curves modeling. J Clean Prod, v. 181, p.145-154, abr. 2018.

INTERNATIONAL Agency for Research on Cancer. Coffee, Tea, Mate, Methylxanthines, and Methylglyoxal; World Health Organization (WHO): Lyon, France, Vol. 51, 513p, 1991.

MOHAMMADI, Shohreh et al. Phenol removal from industrial wastewaters: a short review. Desalination And Water Treatment, v. 53, n. 8, p.2215-2234, 30 jan. 2014.

MOHAN, D.; PITTMAN JR., C.U.; STEELE, P. H.; Pyrolysis of Wood/Biomass for Bio-oil: A Critical Review. Energy \& Fuels, v. 20, p.848-889, 2006.

NASCIMENTO FILHO, V.F. Fluorescência de raios X por reflexão total: fundamento e aplicações. Piracicaba: CENA/USP, p.93, 1997.

SANTOS, S. S. dos et al. Adsorção de fenol e benzeno em montmorilonita modificada com brometo de hexadeciltrimetilamônio. Cerâmica, v. 60, n. 356, p.575-579, dez. 2014.

SILVA, C.G.A. e COLLINS, C.H. Aplicações de cromatografia líquida de alta eficiência para o estudo de poluentes orgânicos emergentes. Quím. Nova, Vol. 34, No. 4, 665-676, 2011.

SKOOG, WEST, HOLLER, CROUCH, Fundamentos de Química Analítica, Tradução da $8^{\mathrm{a}}$ Edição norte-americana, p.678. Editora Thomson, São Paulo-SP, 2006.

WANG S., et al. Removal of arsenic by magnetic biochar prepared from pinewood and natural hematite. Bioresour. Technol. 175, 391-395, 2015.

ZHANG N, et al. Diclofenac photodegradation under simulated sunlight: Effect of different forms of nitrogen and kinetics. Journal of Hazardous Materials 192: 411-418, 2011.

ZHANG, M.; et al. Self-assembly of needle-like layered double hydroxide (LDH) nanocrystals on hydrochar: characterization and phosphate removal ability. RSC Adv., v. 4, p. 2817128175. 2014. 